\title{
Handling Transition from Legacy Aircraft Communication Services to New Ones - A Communication Service Provider's View
}

\author{
Frederic Durand and Luc Longpre \\ Société Internationale des Télécommunications Aéronautiques (SITA) \\ France and Canada
}

\section{Introduction}

The internet hasn't just changed the way we communicate - it has irrevocably altered the way we live, work, consume and spend our free time. Now, technologies that use the internet protocol (IP) in the air transport industry will lead to a similarly dramatic transformation - this time to aircraft operations, whether on the ground or in-flight. Nowhere will this transformation be more evident than the way aircraft communicate. Passengers are already benefitting from this revolution: Passenger connectivity systems already provide internet access, cellular telephony communications while aircraft is in flight.

Today, most options for onboard and external data communications offer limited capacity and versatility. This explains why the amount of information that can be exchanged is restricted to short messages, mainly in predetermined formats. It also explains why a proportion of communications are still conducted over voice. What's more, while the plane is on the ground, there are currently only limited ways of cost-effectively transferring large amounts of information and many of these involve manual downloads and physical storage media. Such constraints have had a major impact on aircraft operational efficiencies and the ability of airlines to automate practices around the aircraft. But the introduction of 'ITenabled aircraft' - sometimes also called 'e-enabled' or 'digital aircraft' - enables secure IP communications to and from the aircraft. This is a critical game-changer for the industry. As the first step towards implementing a complete IT infrastructure in the aircraft, it is set to have a major transformational impact on the way airlines operate their aircraft - not only in the cockpit, but also in terms of cabin procedures, aircraft turnaround, maintenance and passenger services. The impact of the IT-enabled aircraft will be all-pervasive, providing the industry with the means to tackle long standing areas of operational inefficiency.

With IP-enablement, we will see new levels of automation and efficiency in cockpits and cabins, enabling crews and passengers to have access to high speed networks and communications. This paves the way for the introduction of new systems, applications and tools on board the aircraft. The reality is already dawning, with the Airbus A380 and new variants of the Boeing 777, as well as with the impending arrival of new types of aircraft such as the Boeing 787 and the Airbus A350. 
In the collective mind's eye of the air transport industry, IT has rapidly become a facilitator and catalyst for ongoing aircraft development and optimization efforts. While the potential for innovation may seem boundless, our industry requires a profound convergence at many levels - of standards and regulations, collaborative approaches, infrastructure deployments, and more. Only then can all stakeholders' profit from this digital revolution by enhancing their business efficiencies through a new generation of aircraft operations.

In this chapter, we will first go through an overview of the legacy aircraft communication systems and applications, as well as the existing aircraft IP connectivity solutions, in aircraft operations field up to passenger communications. Service providers' missions and positioning will be outlined. Future communications systems and applications, as envisioned by European (SESAR initiative) and US (FAA NextGen) bodies, will then be presented. Possible scenarios in the role and scope of service providers will also be sketched, with a special focus on the integration/emergence of various service fields, ranging from aircraft operations to passenger-related services. The role of Air navigation Service Providers (ANSPs) and relationship with traditional aeronautical communication service providers will be addressed. Also relationship with communication service providers that are newcomers in the aeronautical market will be analyzed. Then a case study based on AeroMACS (Wimax in Aeronautical band) will be presented: the way the system could be operated, identification of ground providers and interactions between these providers, will be presented.

\section{Current and emerging aircraft communication applications and related systems}

As defined per ICAO (International Civil Aviation Organization), Datalink services can be categorized as follows:

- Aeronautical administrative communication (AAC). Communication used by aeronautical operating agencies relating to non-safety communications for the business aspects of operating their flights and transport services.

- Aeronautical operational control (AOC). Communication required for the exercise of authority over the initiation, continuation, diversion or termination of flight for safety, regularity and efficiency reasons.

- Air traffic services (ATS). A generic term meaning variously, flight information service, alerting service, air traffic advisory service, air traffic control service (area control service, approach control service or aerodrome control service).

- Air Passenger Communications (APC), defined as services to passengers providing them an access to communications and entertainment means similar to those that can be experienced on ground.

A number of networks and associated communication means can be used by these Datalink services and applications.

3 types of networks are under operation today in the aeronautical world for aircraft ground communications for cockpit/maintenance/cabin operations:

- ACARS (Aircraft Communications Addressing and Reporting System) used for AAC/AOC/ATC

- $\quad$ ATN (Aeronautical Telecommunications Network) used for ATC

- IP used for AAC/AOC (mostly aircraft IT systems), as well as APC 
Depending on the aircraft type, area of operation, and airline, some or all of the above mentioned network technologies are supported. Traditional Aeronautical Datalink Service Providers (DSPs) such as SITA and ARINC provide ACARS and ATN connectivity services. IP air ground connectivity can be provided by traditional DSPs, or by standard telco operators (e.g. 3G operators). These three technologies will in the frame of future ATN (addressed by EU research SANDRA project), migrate/include an IP connectivity solution for ATN.

Depending on the applications to be supported, standard IP connectivity or aeronautical specific ones with specific Service Level Agreements (SLAs)/ Service Level Objective s (SLOs) will be necessary.

In-Flight Entertainment (IFE) and passenger connectivity services are handled nowadays by a variety of subnetworks, especially new aircraft-ground IP links, as well as specific Satcom Inmarsat services. These will be detailed later in the document.

\subsection{ACARS}

ACARS cockpit data link avionics are installed on approximately 10,000 air transport aircraft and approximately 4,000 business and government aircraft.

ACARS is used by flight operations applications that are hosted in the ACARS avionics unit and is connected to a Multi-Function Control and Display Unit and a cockpit printer that provides input/output to pilots. The ACARS unit is also used as an air-ground router by other airborne systems including the Flight Management system and aircraft system monitoring systems called Digital Flight Data Acquisition Units or Central Maintenance Computers. The ACARS unit communicates with ground networks via various radio systems, always including a VHF radio, and optionally also satellite avionics and/or an HF data radio. Passenger and Cabin application systems can share the use of the satellite avionics if they are installed.

Figure 1 below shows a high level view of end to end ACARS architecture.

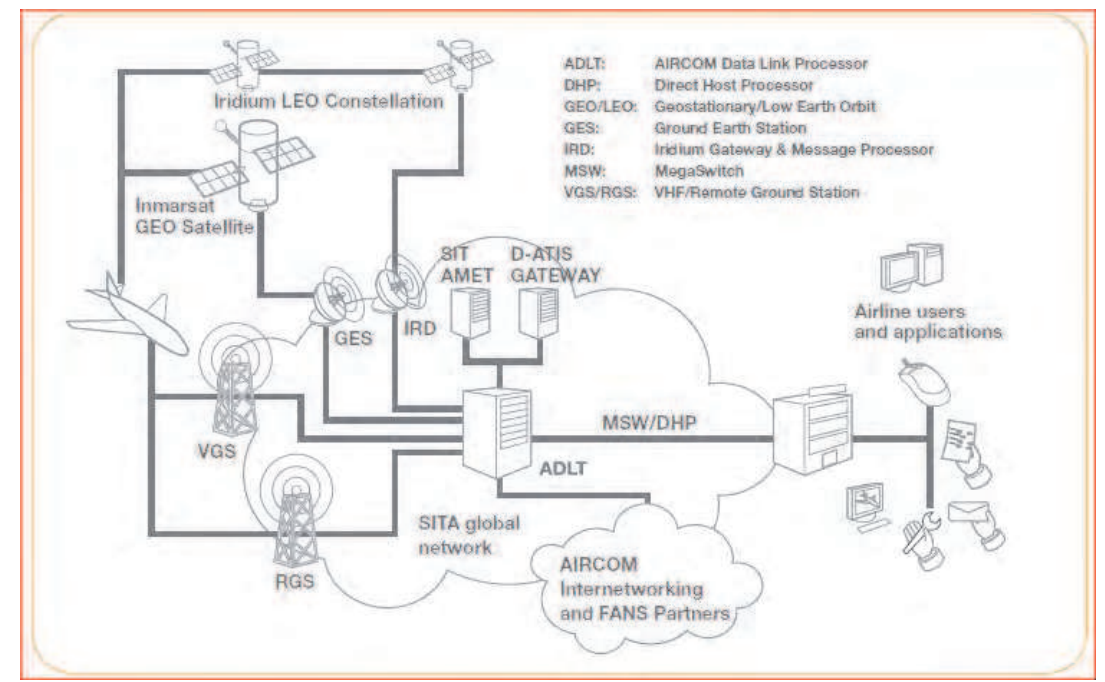

Fig. 1. Overview of SITA ACARS service architecture. 


\section{Subnetworks:}

The following subnetworks are offered by ACARS service:

- VHF:

- $\quad$ VDL mode 1/A (POA : Plain Old ACARS)

- $\quad$ VDL mode 2 (AOA : ACARDS Over AVLC)

- High Frequency Data Link (HFDL)

- Inmarsat Satcom Data2

- Iridium Short Burst Data (SBD)

VHF and HF subnetworks are operated by the ACARS service providers (DSP), while Inmarsat satellite service is operated by Inmarsat (and, depending on the satellite constellation, also with ground telco partners), and Iridium Satellite service is operated by Iridium. In some countries, the Very High Frequency (VHF)/ VHF Digital Link (VDL) subnetworks are operated by the local ANSP (e.g. China...).

\section{Aircraft communications use of Inmarsat satellite links}

Aircraft have been able to carry out voice and data communications via the Inmarsat satellites since around 1990, when these satellites were expanded from their original function of providing services to ships. The number of aircraft equipped to use the Inmarsat aeronautical service today is approximately 2,000 air transport aircraft and another 1,800 business jets or government aircraft. The aircraft using the Inmarsat aeronautical service each month generate a total traffic of approximately 9 million kilobits of ACARS data link messages and 200 thousand minutes of voice calls.

The original Inmarsat Aeronautical service provides two service modes, circuit mode supporting voice communications (or a $2.4 \mathrm{kbit} / \mathrm{sec}$ modem-to-modem data/fax communications) and packet mode supporting "always-on" data communications.

Aircraft equipped with FANS-1/A avionics (ATC safety communications) use this Inmarsat data link service as the primary means of Future Air Navigation System (FANS) communications in oceanic and remote areas. Aircraft operators use the Inmarsat circuit mode to offer telephony service to passengers and to crew in the cockpit and cabin. Aircraft operators use the Inmarsat packet mode, which provides a data rate of up to $9.6 \mathrm{kbit} / \mathrm{second}$, for ACARS communications.

\section{Aircraft data link using HF Radio}

The move of aircraft communications from voice to data has motivated some operators of HF radio ground stations to install HFDL computers that enable them to transport ACARS communications.

The vendors of aircraft HF radios have added corresponding capability to support ACARS and it has been installed by a few airlines. The new HF avionics radios can switch between voice and data mode using the same aerial, but they are required to give voice communications precedence over data link, which limits the HFDL availability. A limited number of aircraft are using HF data link and it has been found to provide better availability than HF voice on the routes over the Poles beyond the 80-degree North/South limit of Inmarsat satellite coverage. The HFDL capacity is limited by the frequencies available in the HF band. The allocation of HF frequencies to data link has required a very complex coordination process and the system will quickly reach the limits of available capacity. HFDL subnetwork has been also qualified for use for ATS communications. 


\section{ACARS Iridium satellite air-ground link}

Since 2007 ACARS avionics have begun to be linked to avionics that use the Iridium satellites which fly in low earth orbit and allow avionics to be lighter and less costly. These ACARS messages are being sent in Iridium SBD transmissions. SITA has implemented a gateway between the ACARS service processor and the Iridium SBD server to provide the service via Iridium. Iridium SBD service is being qualified for use for ATS communications.

\section{ACARS over VDL}

The air traffic control community defined the ICAO VDL standard to transport ATN airground communications but ACARS communications can also use the VDL link. Following discussion of the options for ACARS use of VDL, the Airlines Electronic Engineering Committee (AEEC) Data Link Users Forum in January 1999 adopted as the standard interim architecture "ACARS over AVLC" (AOA).

In the VDL AOA architecture, aircraft use the AEEC 618 protocol over the ICAO VDL standard AVLC link providing 31.5-kilobit per second capacity. Aircraft using VDL AOA obtain increased capacity over the VHF link but can only exchange messages in the same ACARS AEEC 618 formats used over the existing VHF analog link.

\section{ACARS service}

The ACARS messaging service is supported by the DSP, and implemented by a set of resilient ACARS message processor(s) (ADLT), with management systems. Internetworking between DSPs is possible, mostly for ATC services.

\section{Who operates and what are the business relationships?}

Historical Datalink Service Providers provide ground-ground ATI messaging (TypeB) as well as Aircraft to Ground ACARS messaging. ARINC and SITA have been providing these services for tenth of years now, also with Type A services (aircraft-ground communications). VHF ACARS was the first communication technology developed, later followed by Inmarsat Satellite link. For VHF, several national operators have also been present in this market in a situation of monopoly (China, Japan, etc.). ARINC and SITA have developed internetworking agreements with these local operators to extend their services to these countries.

In early 2000, both ARINC and SITA obtained the right to deploy VHF in the traditional coverage domains of their competitors, i.e. SITA in North America, and ARINC in Europe. This resulted in a service availability improvement (as very often, airlines contract one service provider (primary), as well as a back up, to improve VHF service availability), as well as prices reduction. Only ARINC and SITA provide an ACARS service using satellite communications. ARINC is the only provider of an HF Datalink service for ACARS. SITA relies on Iridium to provide an equivalent coverage (polar routes especially). ACARS supports ATC, AOC, and AAC communications, not IFE/Pax communications, as per regulations.

Ground actors in their strong majority use private network solutions to access ACARS service to aircraft. This is the only reasonable way to meet end to end service level objectives. In a few cases, internet can be used for front end connectivity access to ACARS service, but for non safety / availability application types. It may also be used for downlink only traffic (aircraft to ground). When ATC/AOC come into play, private networking solutions are deemed inevitable by stakeholders to reach adequate security and guarantied performances. For the same reason, we expect that such communication over IP network will also requires private predictable ground networking for these applications. 


\subsection{ATN/OSI}

\subsubsection{ICAO VHF air-ground digital link (VDL) mode 2}

The ICAO VHF Digital Link (VDL) Mode 2 standard was developed following the 1990 ICAO Communications Divisional meeting that recognized the value of specifying the use of the Aeronautical VHF channels for data communications. The 1990 ICAO Communications Divisional meeting also reserved the 4 channels 136.900, 136.925, 136.950 and $136.975 \mathrm{MHz}$ for data communications worldwide. Following that meeting, the ICAO Air navigation Commission created the Aeronautical Mobile Communications Panel (AMCP) to develop the VDL standard. The validated VDL Mode 2 standard was presented to the AMCP at its fourth meeting in March 1996, which recommended that it be included in Annex 10. The ICAO member states accepted this recommendation by agreeing to its inclusion in Amendment 72 to Annex 10.

The ICAO VDL Mode 2 standard specifies the use over the VHF link of a D8PSK (Differentially encoded 8-Phase shift Keying) modulation scheme providing a data rate of $31.5 \mathrm{kbits} /$ second compared to the VHF ACARS rate of $2.4 \mathrm{kbits} / \mathrm{second}$ in the same channel width of $25 \mathrm{kHz}$. The VDL Link Layer protocol specifies for media access control to the VHF channel the same Carrier Sense Multiple Access (CSMA) algorithm as for classic VHF ACARS. However, the VDL CSMA will provide better performance than the VHF ACARS CSMA by using a VHF Data Radio to process the CSMA function. The combination of the VDL D8PSK scheme and its CSMA algorithm makes the link reach saturation at a data load of 10 kilobits per second, compared to the classic VHF ACARS maximum effective link capacity of 300 bits per second.

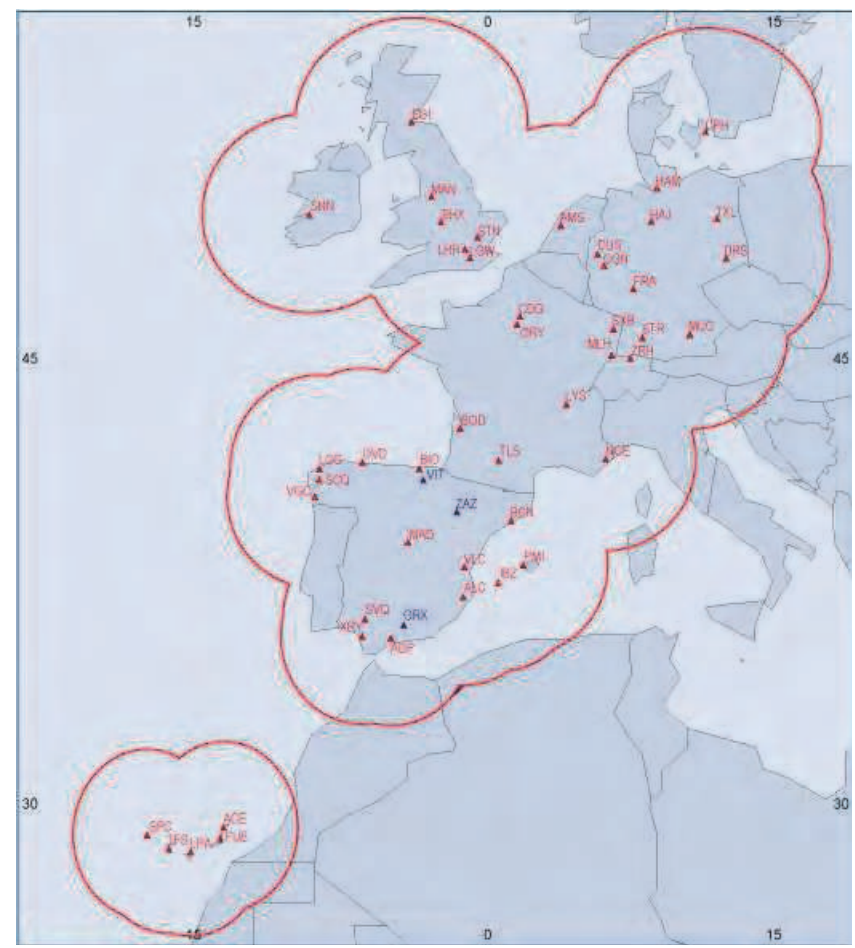

Fig. 2. SITA VDL2 network in Europe. 
The "Aviation VHF Link Control" (AVLC) protocol provides a link for the transport of binary data between an aircraft and a ground station. AVLC is a variation of the ISO High Level Data Link Control (HDLC) protocol, designed specifically to handle the use of VHF channels.

\section{ATN (ATN/OSI) End-to-End Protocols}

The ATN and data link standards specify protocols using the logic and terminology of the International Organization for standardization (ISO) model for Open systems Interconnection (OSI). The ATN standard covers upper layer protocols used in end systems but this document focuses only on the ATN transport and network layer protocols. The ATN standard specifies ATN applications use of ISO 8073 Connection Oriented Transport Protocol (COTP) over the ISO 8473 Connection Less Network Protocol (CLNP). The COTP protocol provides a message delivery acknowledgement over the CLNP protocol which handles the actual message exchange between the ATN users systems. The ICAO ATN standard specifies a unique addressing scheme to be used in the CLNP protocol which has two formats:

- ANSP systems: ISO county code, city code, terminal identifier

- Airline systems: ICAO airline code, city code, terminal identifier

The ATN CLNP messages are handled by routers that interconnect air-ground (mobile) subnetworks and terrestrial subnetworks. The routers establish a routing information base using ATN routing protocols, primarily the ISO 10747 Interdomain Routing Protocol (IDRP). The ATN routing protocols establish a CLNP routing information base, which is updated as the system establishes subnetwork connections to other ATN systems. Airborne ATN routers maintain a routing information base indicating which connections are available over air-ground data link subnetworks.

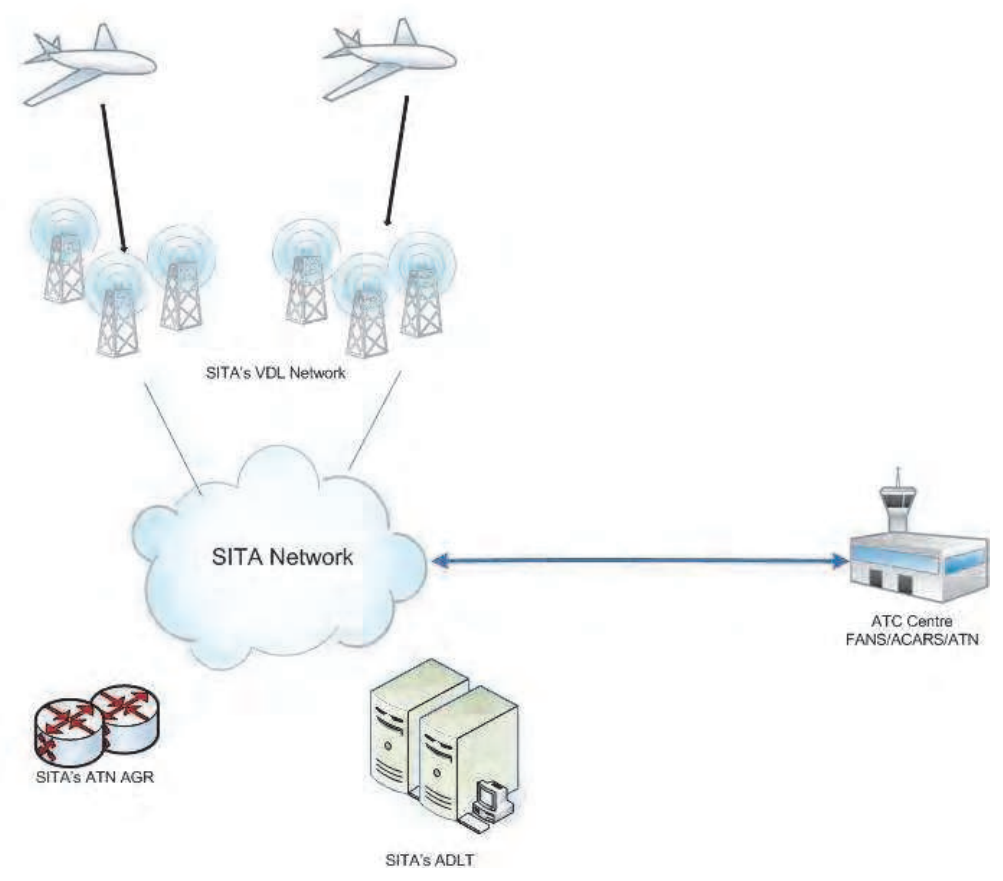

Fig. 3. Overview of SITA ATN architecture. 


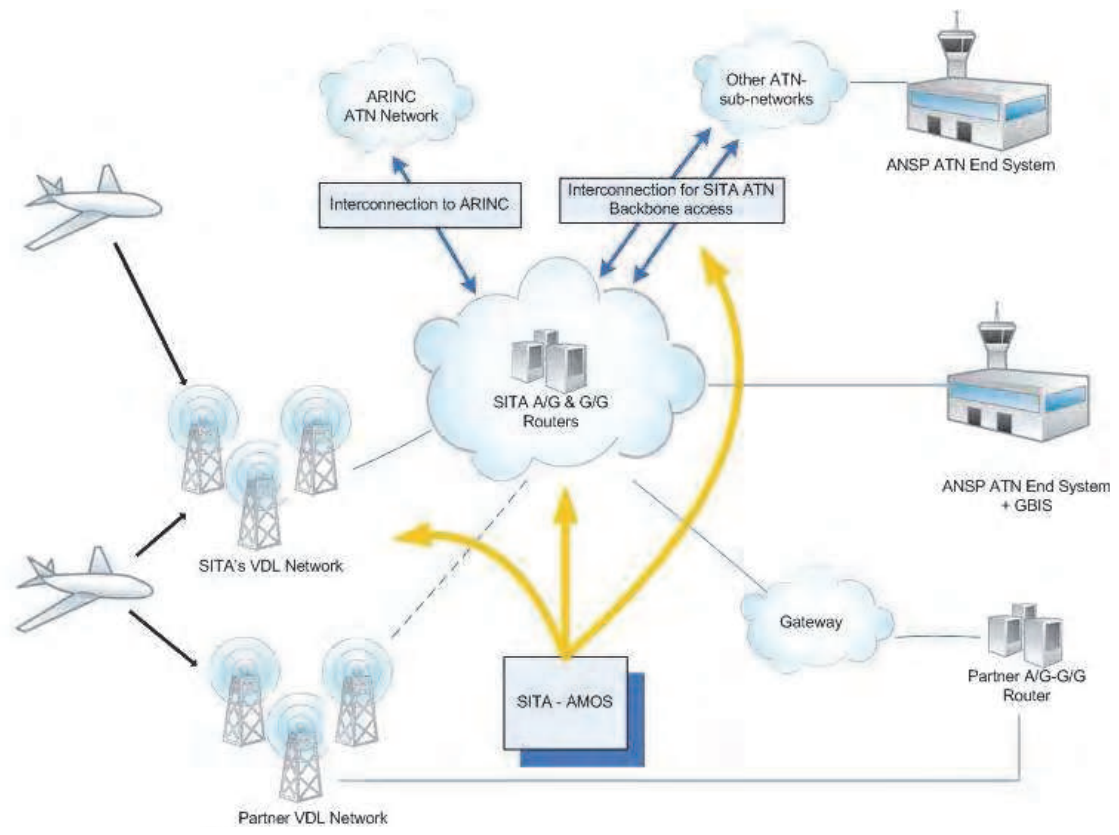

Fig. 4. Overview of SITA ATN architecture - interface with other parties.

It has to be noted that all ATN services can be supported by:

- X25 network infrastructure

- IP infrastructure

Operators are / have migrating to IP (e.g. SITA provides access to AGRs through IP WAN connection (aka IP SNDCF)).

\subsection{Emerging IP connectivity for EFB and cabin}

In analogy to how modern technology changed and improved modern organisation capabilities, airlines are being convinced that cockpit IT systems will enable them to implement more efficient operations. New cockpit IT systems and applications are likely to rely heavily on IP links for operational purposes in ways analogous to how current systems and processes rely currently on ACARS services.

\section{Comparison of IT equipment using IP in the organization Vs in aircraft communication}

Computer have been around for a long time, some of you will remember that hard disks used to be the size of a washing machine and were able to store just a few megabytes of data. Today, people carry in their pockets devices that can hold a thousand times more information. At the same time people were using terminals that could only send and receive 240 characters or 2,400 bits per second as opposed to today's were you can often achieve speed more than 10 mega bits per second using an high speed internet connection.

This is a simple illustration of the technology evolution in the last 25 years. The pace of this evolution as not reduced, on the contrary new technology and new ways to use it evolve at a continuously augmenting rhythm. Each new invention leads to more and more possibilities and also provides the necessary foundations for even more new ideas. 
Considering this evolution, the assets used in general Information Technology systems (IT) are subject to frequent changes, shorter duration and amortization. In contrast, technology assets in the aerospace industry typically have very long economic lives and are consequently not usually managed in the same way as general IT assets. Many systems that are delivered in today's aircrafts will remain the same in 20 years. To illustrate the reality of this, we can mention that today's modern aircrafts are equipped with ACARS that is able to transfer 2400 bits per second or about only 10 times more with the newer VHF DataLink Mode 2. This is similar speed to the above 20 years old terminals which cannot be seen anymore in any modern organization. In a recent survey done with New Generation Aircraft (NGA) current and future operators, they have clearly indicated that ACARS will remain an important component of the aircrafts communication infrastructure for the foreseen future.

Although we cannot expect the same lengthy lifespan with new IT systems that gets installed in aircrafts, we can certainly expect that if a technology solution reaches a critical mass of installed based in aircrafts, its corresponding ground infrastructure and associated operational practices, that such solution may certainly last longer in the Air Transport Industry (ATI) then in its counterpart in the general IT market. In the same survey mentioned above, an interesting statement was made about selecting the right technology for a large retro-fit program:

- The time required to accomplish a fleet-wide implementation is longer than the expected life cycle of current solutions. - "We have a vision, but will it be the right one when we have delivered it? We like the technology and we want to use it, but I fear our choices now will be old when the project is fully rolled out".

In consequence, we could anticipate that if airlines and service providers reach a point where massive deployment can be made that the selected solution will last for many years. In addition if as mentioned above this solution life cycle is somehow longer when used in the ATI, that a specific ecosystem will have to exist to maintain it.

\section{What will be this solution? Will this ever happen?}

In the next paragraphs we will elaborate on the various conditions and elements that affect the eventual choice and potentials for some sort of industry solution to reach the needed critical mass that will certainly affect the way airlines operates their fleets.

\section{Drivers for new IT systems}

In general, key drivers for airlines revolve around attracting and retaining customers; efficient management and operation of their fleets; improving personnel and asset productivity; maintaining safe operations and managing their financial cycles. New cockpit IT systems affect the way aircraft are operated and maintained, so improvements in these areas can result in increased productivity and lower costs

In substance, the main drivers that leads airline to use the capabilities provided by the new technology are the same that drives any other projects:

- $\quad$ Streamlined Processes

- Operational Efficiencies

- Cost Control \& Reduction.

The deliveries of new aircrafts such as the A380 and B787 that come equipped with new IT systems are also leading airlines to looks at ways to use these to achieve the above objectives. 
Some important barriers need to be considered before projects get implemented:

- The first one, and probably the most important one: The business case that would justify the necessary investment in a major project, its required communication capabilities and necessary enhancements to operational practices is not an easy one. Cost saving and production improvements although perceived as obvious when thinking about the use of modern IT systems and high speed IP communication becomes less obvious when all organizational costs and impacts of a project are added up.

- Typically the IP broadband capabilities of the links available for aircrafts are often justified by the cabin needs, yet it becomes difficult to justify their full use for cockpit operational needs. This lead to the need of cross organizational programs, which are not tradition in a typical airline. So time \& effort is required to reach the necessary level of internal co-operation,

- The technology choices have a large impact on costs and no technology solution as yet reached a massive number of aircrafts to be considered as a no-risk choice.

- Security has been high on the radar. However it has been thoroughly addressed by the experienced players, thus security is not seen as a big problem, as long as it is addressed with rigour.

It takes a forward-thinking planner smart enough to envision a way to use the new technology successfully in the design and operation of a brand new aircrafts. Very often successful implementation of new systems and the necessary operational changes would require fleet-wide adoption to get the full benefits. So not only using new systems in new aircrafts conditions the success of a program but consideration to adopt fleet wide systems and processes also become important.

Airlines with multiple fleets will also require assistance to reduce the complexity and differences to deploy maintain and support the various ground systems and communication links required to manage the various IT solution proposed by the air framers and other solution available in the retro-fit market. In the long run, it is expected that airlines will attempt to eliminate dissimilar operational processes and systems across their fleets .This assistance will be provided by their selected suppliers and partners.

As viewed above when choosing a solution, airlines have to consider the solution expected life-time and select the communication technology that will provide the required global geographical coverage at the right performance and right cost. Otherwise they may be eventually at risk of having to support by themselves the entire ecosystem of the selected solution. In such condition, the expected benefits of the new IT systems may not be as profitable as originally expected.

As a consequence of the difficulties to implement new innovative projects using new technology in aircrafts, many airlines that currently operates new generation aircraft that are provisioned for or equipped with wireless IP avionics connected to Cockpit IT systems make limited used of the capabilities at their hubs only an often not at all.

Only very few airlines are currently planning to use new broadband capabilities outside their hub airports or major stations; however, it is expected that the initial delivery of the Boeing 787 now planned for 2012 will bring more opportunities for changes. In addition former manual processes might not even be possible anymore due to turn around constraints and data volumes. Similarly the entry into production of the Airbus A350 (planned in 2013) might also bring significant business incentives to implement new practices relying on broadband IP wireless systems. 
Considering the above, airlines have to be careful when considering their choice of partners and suppliers and look for the ones who understand the complexity of airline operation with mix-fleets and that are expected to remain strong players in the ATI for the foreseen future. The current financial status of these organizations as well as their past history would also be a good indication that they can be an adequate choice.

\section{Benefits of using commercially available Off-The-Shelf (COTS) technology}

Aircraft undergo severe conditions in their regular journey, with frequent and important changes in temperature and atmospheric pressure. In consequence, systems that must be installed aboard an aircraft need to be designed with the aircrafts particular constrains, needs for security, stability and durability. This lead to careful validation, tests and certification while augmenting the development process complexity.

While using Commercially available Off-The-Shelf (COTS) IT technology and protocols instead of technology that is particularly built for aircrafts may reduce the development cycle, it should be expected that all other particular requirements remains part of the design objectives. As such only marginal saving should be expected to equip and maintain the new IT systems installed aboard the aircrafts.

Certain confusion can be observed in the market with the air framers introduction of COTS IT systems in new generation aircrafts. As the technologies used in aviation applications move from purpose-built to generic, the entry barriers for new entrants have been considerably lowered. Many organizations who historically have not served the ATI industry are now seeking opportunities to extend their market to this field simply based on the fact that the same technology they are familiar with is starting to be used in aircrafts. As seen above COTS IT is only one factor, and not the most important one that must be taken into consideration to adequately serve this particular industry.

The sum of solutions proposed to airlines is so large that no single technology, systems and IP broadband connectivity is yet rising to become an industry standard. While this allows for strong differentiation between airline offers, this also prevents reaching a critical mass that would drive costs and price down for the benefit of everyone. It is important to note that all IT systems installed aboard the aircraft also require their ground counterparts: The back office airline operation systems and at least the global communication infrastructure supporting the chosen aircraft communication method. Massive investment in large and global ground infrastructure will only become possible once a few technology solution raises to become a preferred choice. In the mean time the industry is left with a ground infrastructure that offers disparate coverage, limited to certain region or airports.

Airlines will be looking into achieving the following as listed in Table 1 with the introduction of the new IT systems.

\section{The players}

Who can play a role in deploying infrastructure suitable to provide and support new aircraft IT systems and associated ground components?

- Airport can provide local IP broadband connectivity to their hub airlines. This is reality already. This is typically Wi-Fi connectivity from the gate, hangars to the aircraft;

- Airports may also extend their service offering to other airline flying to their facilities. This may work quite well for a few airports and airlines, but will certainly fail attempting to extend the model: Each airline would require entering into a specific and often complex project with many airports to get the necessary connectivity at multiple 
outstations. Such task is viewed by many airline as too complex and too costly to be seriously considered on a wide scale;

- Cellular provider with their continuously increased network performance and decreasing prices are already common provider of IP broadband connectivity solution for aircraft. While this model currently works best in the provider local country, the roaming model is less attractive with higher prices. Solutions to these high roaming costs are rising: using device that can support multiple provider SIM cards along with the necessary technical capability that allow choosing the right SIM card base on current location and;

- Global SIM card providers who can negotiate very competitive pricing with multiple providers based on volume and usage projection. These last types of offers, although very recent, seem to be a good model for aircrafts that need to travel in multiple regions. One other issue that may arise with this technology and its communication method is the fact that the cellular networks are usually shared by many users and may suffer from congestion problems. We suspect such problems to rapidly fade away as cellular providers often have the right business justification with the increasing volume of users to invest in enhancement of their networks. Global SIM card provider may also benefit from being already able to offer network connectivity using dedicated circuit which allow offering services with the level of performance and stability suitable for airline operational activities while not suffering from the congestion problems observed with local cellular providers;

- Global Satellite provider such as Iridium and Inmarsat offer IP broadband service through distribution partner mainly for in-flight connectivity. Each major provider has offers that competes and have gained some market share.

- Global datalink service providers already servicing the ATI, mainly SITA and ARINC are developing solution to address the IT systems and connectivity needs of the new aircrafts systems.

- New entrant in the ATI industry. The introduction of cockpit IT brings new opportunities to companies currently absent in the aircraft communications and connectivity market but who may have experience integrating and offering groundbased broadband and IP solutions. Many are looking to complement their existing portfolios and move into aircraft IT systems and connectivity. In addition the availability and commonality of IP-based network connectivity through increasingly accessible satellite operators and with the Internet reduces necessary investments in some infrastructures to offer solution that may win some of the aircraft IT and connectivity business.

As of now the industry in general, benefits from a mass of expert in some of the common aspect of the systems that gets installed in aircraft and in particular, the IP protocol used by these systems. Considering the difference in life cycles of the technology assets that gets installed in the aircraft and the IT industry in general, this mass of expertise might only be a temporary situation lasting only until IT systems reach a new level of sophistication. We may reach a time where the general perception is that aircraft IT systems are archaic, and only perceived as fit for this unique vertical market, very similar to today's situation with legacy aircraft systems. In consequence, airlines when selecting their aircraft IT suppliers must be careful to consider their overall ATI expertise and their real chance to last in this particular industry. 


\begin{tabular}{|l|l|}
\hline \multicolumn{1}{|c|}{ Expected Benefit } & \multicolumn{1}{c|}{ Supporting Features } \\
\hline $\begin{array}{l}\text { Cost savings and } \\
\text { operational improvements } \\
\text { through better and greater } \\
\text { access to aircraft and post- } \\
\text { flight data while on ground }\end{array}$ & $\begin{array}{l}\text { Wireless broadband access to aircraft and flight data upon } \\
\text { landing during turnarounds and layovers } \\
\text { Use of low cost wireless technology to transfer large } \\
\text { amounts of non-critical data (trending, manuals, etc.) }\end{array}$ \\
\hline $\begin{array}{l}\text { Flight operational } \\
\text { efficiencies achieved } \\
\text { through access to most } \\
\text { recent data prior to } \\
\text { departure }\end{array}$ & $\begin{array}{l}\text { Ability to send flight crews latest information while at the } \\
\text { gate. }\end{array}$ \\
\hline $\begin{array}{l}\text { Process automation and } \\
\text { labor cost reductions in low } \\
\text { value activities }\end{array}$ & $\begin{array}{l}\text { Wireless data transfers represent a vast improvement over } \\
\text { manual data retrieval and documentation updating } \\
\text { processes } \\
\text { Resources and time can be shifted to higher value, more } \\
\text { productive activities }\end{array}$ \\
\hline $\begin{array}{l}\text { Aircraft as a flying node in } \\
\text { airline IT infrastructure }\end{array}$ & $\begin{array}{l}\text { Seamless integration to back-office and 3rd party systems } \\
\text { and processes to enable differentiation, cost saving and } \\
\text { revenue generating initiatives. }\end{array}$ \\
\hline Secure connectivity & $\begin{array}{l}\text { Ability to offer a flexible yet secure environment } \\
\text { specifically tailored to address the needs of aircraft } \\
\text { communications over IP }\end{array}$ \\
\hline $\begin{array}{l}\text { EFB usage reduced } \\
\text { operational costs/Savings }\end{array}$ & $\begin{array}{l}\text { Aircraft use of Electronic Flight Bags (EFBs) using IP } \\
\text { communications will allow them to reduce the amount of } \\
\text { paper needing to be provided to pilots and processed } \\
\text { manually after the end of flights enabling airlines to save } \\
\text { money }\end{array}$ \\
\hline $\begin{array}{l}\text { Saving are then based on crew's productivity gains and } \\
\text { better flight decision due to more accurate and up-to-date- } \\
\text { information as well as a possible reduction in weight (no } \\
\text { more paper to carry). }\end{array}$ \\
\hline $\begin{array}{l}\text { Airline installation of new IT systems, IP communications } \\
\text { and operational practices will enable them to be more } \\
\text { efficient than their competitors }\end{array}$ \\
\hline ingrovements advantage
\end{tabular}

Table 1. Overview of benefits expected from introductyion of new IT systems.

It should also be expected, in the next few years, a high rate of adjustments in this market with strong consolidation of the various offers and many partnerships between software shops, avionics vendors, service providers and others.

\section{Who want to play in this game?}

In general, multiple technologies have proven their technical validity to provide high speed IP connection to aircrafts. Most should be capable of supporting both cabin and cockpit communications (even if they currently don't):

1. Based on ground wireless network:

- Generic 3G cellular network for ground communication 
- Wi-fi with the Gatelink solutions;

- Cellular network directed toward aircraft such as the Aircell solution operating in the US.

- Wimax that is being introduced by a number of vendors

2. High speed geostationary satellites:

- Ku-Band

- Inmarsat new I-4 constellation of satellites

3. Low orbital altitude flying moving satellites

- Iridium constellation of 66 satellites that can support data service up to $128 \mathrm{Kbps}$

- Iridium next generation satellite network, NEXT, planned for 2014/15

Each technology has its merits and limitations. As such it is expected that most will be in the market for a number of years to come. What is less certain concerns the right commercial approach to develop and gain market share. Regulatory aspects are also important, and they will also affect the adoption of one technology over another for cockpit communication.

Today no standard approach has been adopted by airlines to implement new practices and infrastructure to accommodate broadband aircraft communication capabilities. Essentially, each implementation of supporting infrastructure has been unique. The only common IP broadband technology installed in today's major airframers New Generation Aircraft (NGA) is the availability of a Terminal Wireless Lan Unit (TWLU) capable of wireless IEEE 802.11 connectivity from and to cockpit systems. Cellular connectivity is also widely used, but is not generally connected to cockpit systems other than to the Quick Access Recorder (QAR). EFBs are the main type of cockpit IT systems in use today. They require a level of resilience above that needed by the non-critical applications, but the data exchanges, at least initially, still can be limited to hubs and main stations; however the increasing complexity of EFB applications will also make the IP wireless links (whether in the hubs in flight or at out stations) and overall connectivity to airlines own networks much more critical for airlines operations.

In addition to the normal use of ACARS for in-flight AOC and ATS communications, NGAs will use more and more IP-wireless links not only to refresh the contents of their EFBs and IFEs or to download massive amounts of flight and aircraft-related information, but also to upload critical software parts and to access third parties' networks while on the ground. The availability and coverage of IP wireless links will shortly become paramount for airlines operating these aircraft types. As of now most airlines have selected to use the wireless IP broadband link at their hub only but the increase fleet size and requirement to cut costs and increase productivity, is starting to trigger projects that aims to use this same capability at out-stations. As such service providers will shortly be under considerable pressure to provide IP-based services and coverage to facilitate the dispatch-ability of the aircrafts regardless of where they fly.

The upcoming Boeing 787 will bring a marked departure from other NGA already delivered. In fact current Boeing 777 and Airbus A380 operations do not imperatively require IP broadband wireless connectivity. Data transfer can be deferred to hub-only operations.

As mentioned before, the 787 fleets with its increased complex IT systems will bring more opportunities for changes, since the volume, frequency, and criticality of exchanges of operational information between the aircraft and ground systems is expected to be higher than for older aircraft. It is expected that without the use of wireless links such as GateLink, 
to transfer 787 data prior or after every flight that airlines may run into operational inefficiencies. Consequently early 787 customers are expected to lead the way in their adoption of new operational practices and systems surrounding aircraft connectivity, and they form the primary initiators and requester of changes that may lead to a wider scale industry adoption of standard solutions than what we have seen up to now. The same can be said about the next up-coming new fleets such as the Airbus A350.

As an example to illustrate this need of increase data exchange for new aircraft is the Quick Access recorder (QAR) data, known in the 787 as continuous parameter logging, or Continuous Parameter Logging (CPL) which could produce up to $100 \mathrm{MB}$ per flight. This can take a considerable amount of time to download manually, and could be lost if the transfer is not completed before the system memory is exhausted and overwrites the earliest data. The same may apply to the engine health monitoring (EHM) data.

All other aircraft types (including 777s and A380s) can now be handled using legacy services and practices, so there are presently limited incentive or urgency for undertaking the significant investments to install or use new wireless technology even if the IT systems installed in these aircraft allows such use.

In resume expectation are that early Boeing 787 operators may lead the way in their adoption of new operational practices and systems surrounding aircraft connectivity.

\section{Future communications systems and applications}

The future SESAR ATM concept demands datalink services supporting features such as 4D trajectory management, ASAS separation, automation, and SWIM. A reliable and efficient communication infrastructure will have to serve all airspace users in all types of airspace and phases of flight, providing the appropriate Quality of Service needed by the most demanding applications. The mobile part of this infrastructure will be based on a multilink approach, composed of three different subnetworks:

- LDACS: A ground-based line of sight datalink as the main system in continental airspace and supporting Air/Ground services and possibly Air/Air services, offering a high Quality of Service which will be necessary in the high density areas; two systems are under consideration (LDACS 1 and 2) with the objective to select one for implementation. Both operate in the L-Band and are based on modern and efficient protocols;

- Satellite: A satellite based system providing the required capacity and Quality of Service to serve oceanic airspace whilst complementing ground-based continental datalink as a way of improving the total availability. The system is being defined in close cooperation with the European Space Agency. The type of satellite constellation to be used (dedicated or commercial) is still under consideration;

- AeroMACS: A system dedicated to airport operations, based on mobile Wimax 802.16e, providing a broadband capacity to support the exchanges of a significant amount of information such as the uploading of databases or maps in the aircraft.

In addition, and to allow in the medium term interoperability with military operations, a gateway is being defined to interconnect the ATM system and the military link 16 system. Several research programmes have been launched to define, develop, and validate these new solutions, and prepare the Aeronautical community to transition to these new access networks. These activities are handled within SESAR programme. The SANDRA project also takes into account the integration aspects of these new solutions, and the networking environment (IPv6 will be introduced in place of IPv4). 
Figure 5 gives an example of various networks and consequently operators that could be involved in future ATS/AOC communications.

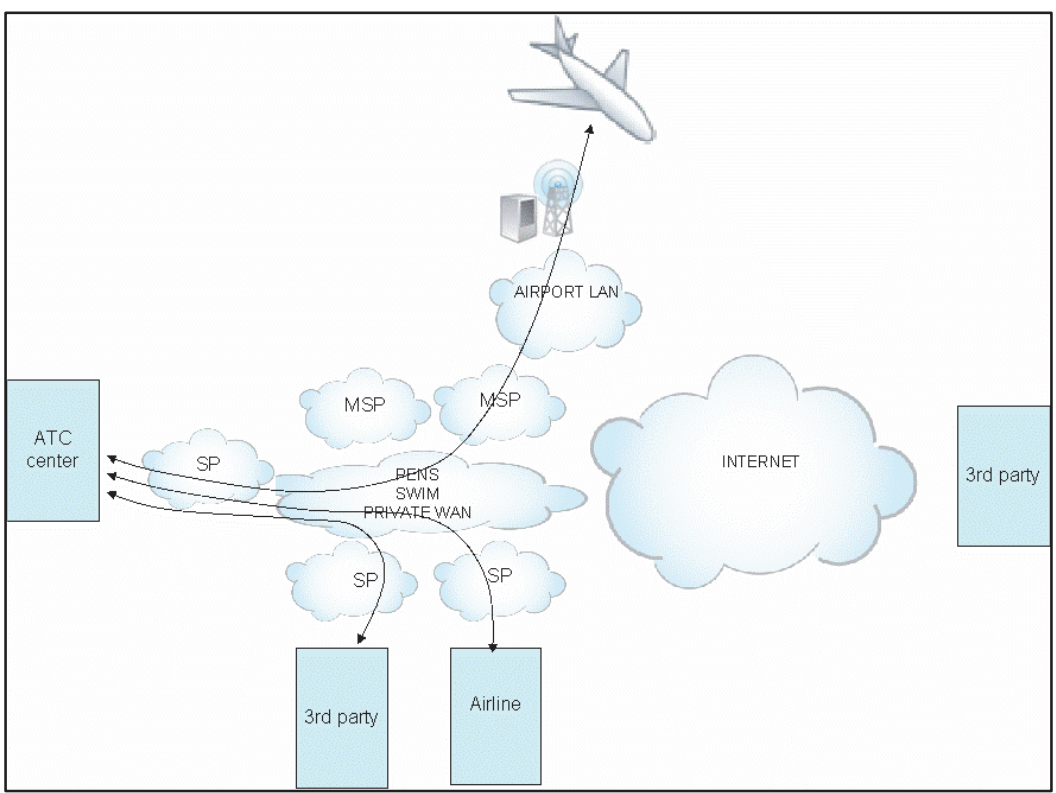

Fig. 5. Example of networks and actors that could be involved in future ATS/AOC communications.

One major difference with IPv4/existing IP connectivity services is that mobility management will probably be provided as a service. Mobile IP being part of the basic scope of future ATS communications. Mobility Service Providers (MSPs) will thus be needed. We can imagine of course having different MSPs between the APC domain and other domains. The service provider on the APC side may even not be aero-specific. However, when we compare these new schemes with the legacy ones (will be detailed in the following chapters), the main actors types remain.

\section{Analysis of service providers' roles and business relationship}

\subsection{Now (ACARS, ATN, IP)}

The ACARS business relationship can be modelled as shown in the diagram of Figure 6 . With a limited number of organizations dealing in this market, the model is very simple.

The actors identified are:

- Users: Airline (Aircraft), ANSPs (Ground ATC End systems), $3^{\text {rd }}$ parties for AOC/AAC

- Global DSPs, providing ACARS service to aircraft and ground access to Aircraft using ACARS service. This is globally limited to two organisations: ARINC and SITA. Global DSP operate also their own VHF/VDL 'almost' worldwide network.

- Local VHF and/or VDL mode 2 operators, providing VHF/VDL2 ACARS service to aircraft, and ground access to global DSPs - a few ANSPs operate their own VDL/VHF network - the trend is however to outsource the network service 

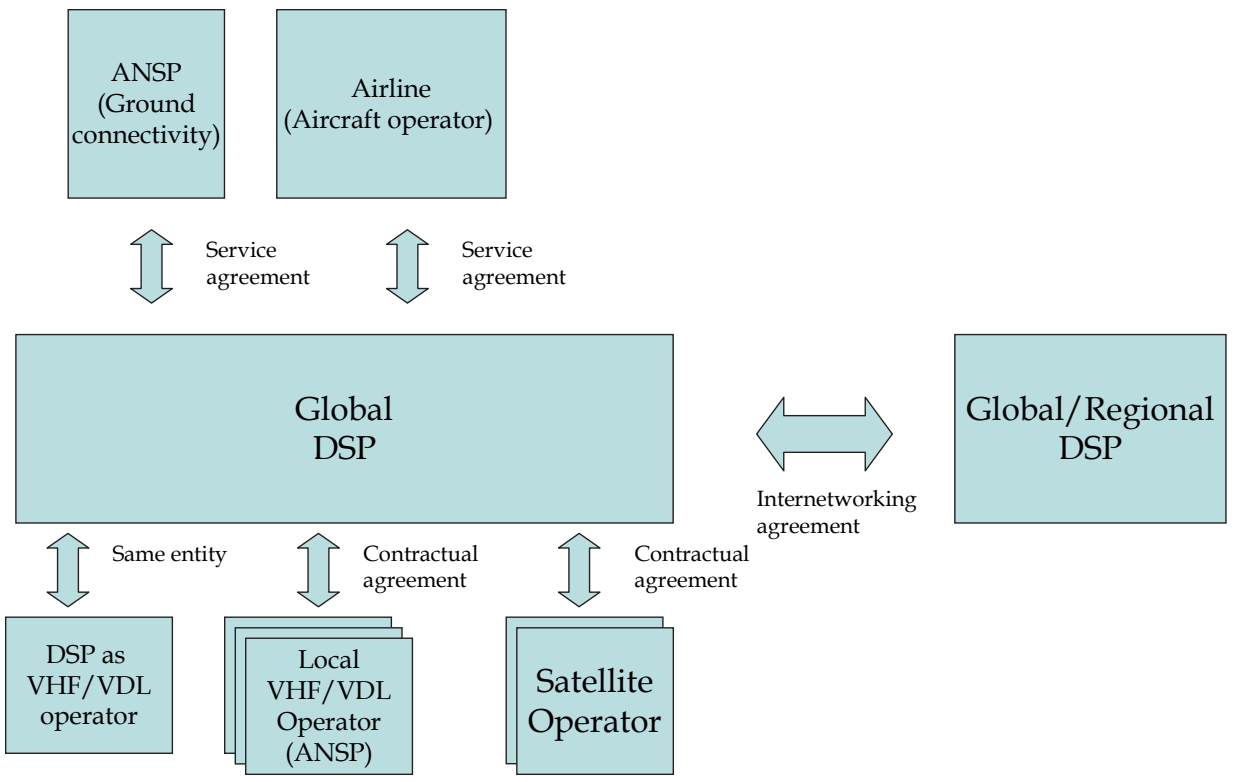

Fig. 6. Illustration of actors' relationship for ACARS.

\subsection{Focus on existing roles and actors in ATN/OSI}

The ATN/VDL2 business relationship can also be simply modelled with a limited number of organizations:

- Users: Airline (Aircraft), ANSPs (Ground ATC End systems)

- ATN operators, providing ATN networking service

- VDL mode 2 operators, providing VDL2 access network and connectivity to Ground ATN network

However, it has to be noted that ANSPs either purchase the VDL2 service to 'global operators' such as SITA and ARINC, or operate the VDL2 service themselves and allow global DSPs to manage the AOC traffic. Even if the overall trend is to outsource such service to partners able to sustain the liability and SLA constraints of safety and dispatch oriented services, these two models will likely be found for future communication means (IP).

\subsection{Focus on new roles with the introduction of new IT systems}

The new business relationships become more complex in the new aircraft IT world with many more players:

- Users: Airline (Aircraft), ANSPs (Ground ATC End systems)

- ATN operators, providing ATN networking service

- VDL mode 2 operators, providing VDL2 access network and connectivity to Ground ATN network

- Global DSPs, providing ACARS service

- Global IP communication service provider

- $\quad$ Regional IP communication service provider 


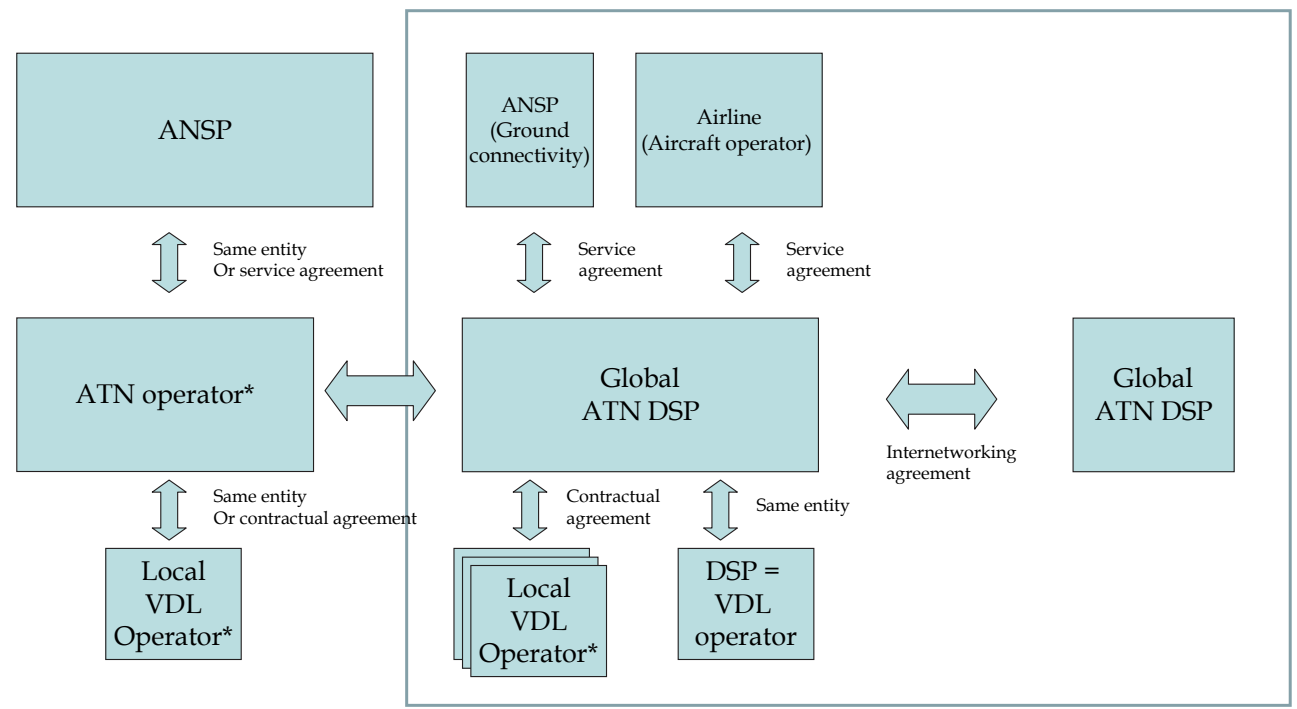

Fig. 7. Illustration of actors' relationship for ATN/OSI over VDL mode 2.

- Local IP communication service provider

- Access network operator (e.g. Inmarsat for SwiftBroadband)

- Solution integrator

- Avionic vendor who now offer multiple IT solutions, communication services and backoffice solutions.

- $\quad$ Airframers IT solutions

- Airports networking services

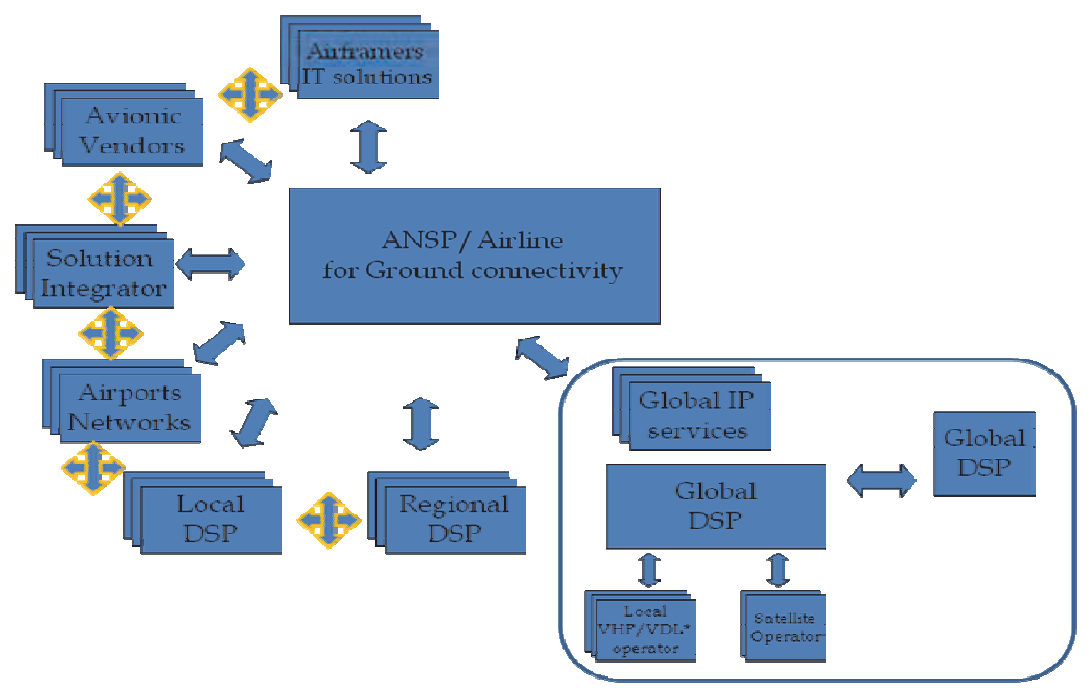

Fig. 8. Identification of main actors for new IT systems operations. 
If we take the example of SwiftBroadband, several major actors are involved

- $\quad$ ASP (application service provider): provides the application using SBB Satcom services

- SP (Service Provider): resells airtime and services to airlines; may activate SBB channel if delegated from DP; may have own APN (Access Point Name: network node on ground for traffic routing), if agreed with DP

- DP (Distribution Partner): SBB channel activation (one SIM card per channel); resells airtime to Service Provider; may directly retail airtime to airline (e.g. OnAir) - DP is linked to SIM card, thus potentially one DP per SBB channel

- $\quad$ Satellite / Swiftbroadband service Operator (Inmarsat)

The actors listed above, specific to SwiftBroadband are Inmarsat and the DP. The other ones can easily perform horizontal integration (with other access networks). A given partnet can perform vertical integration (act as a DP and ASP/SP). All combinations are possible, several DPs per aircraft, several SPs per SIMCard, etc. However, it is of course strongly advised, in order to make the system manageable, to minimize the number of actors and rely on key players.
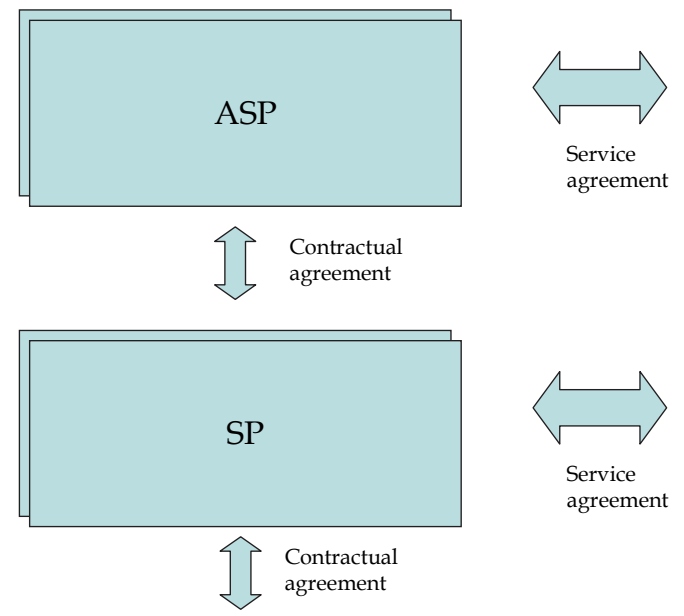

Airline
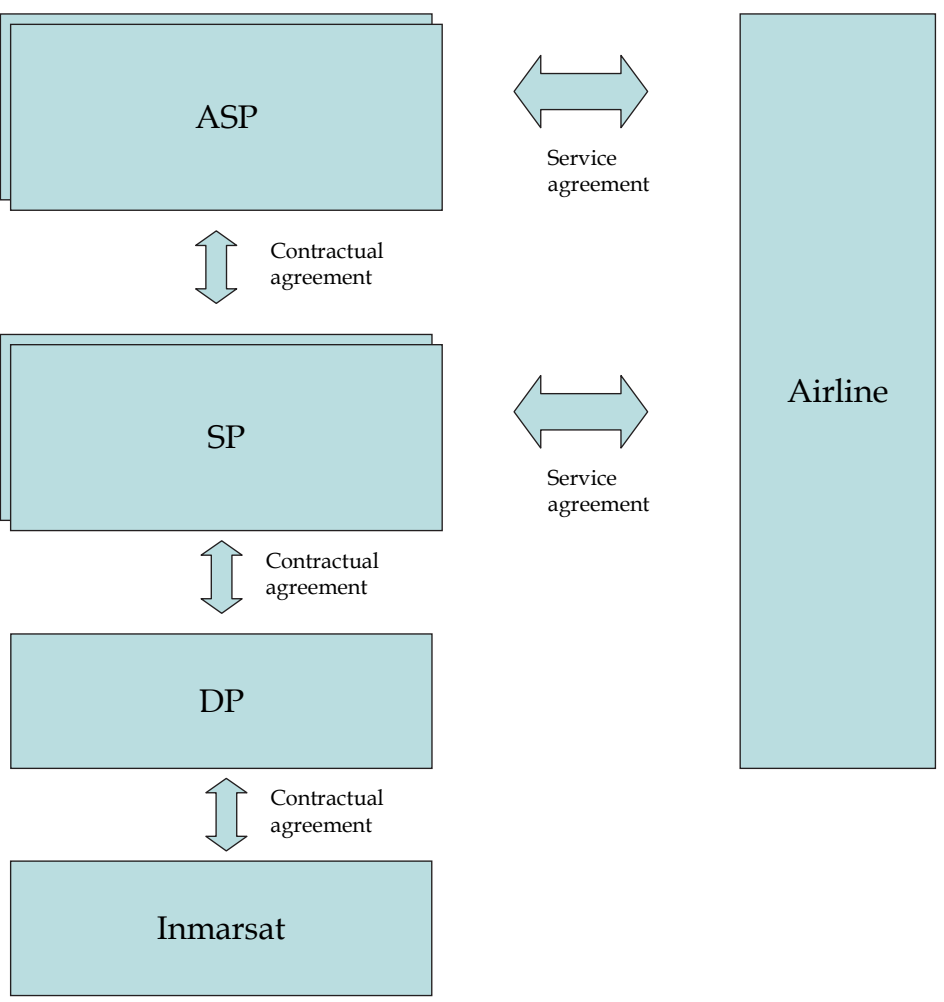

Fig. 9. Actors and relationship for SwiftBroadband 


\subsection{What could be the winning combinations after cards have been shuffled again? 4.4.1 Key technologies and integration on aircraft}

Some of the key enabler to an eventual global successful aircraft connectivity solution, is the availability of adequate aircraft-ground connectivity technologies, at the right performances, with worldwide availability, at the right price and ability to integrate these technologies in a large retro-fit program, at minimum cost and reduced aircraft down time. Security of the solution is also imperative.

Such solutions must then:

- Provide sufficient coverage/availability (regional, airports, etc.)

- Be implementable at minimum cost on aircraft or be provided as part of wider system

A number of proprietary solutions such as Aircell, Teledyne WGL/QAR exist and reached an interesting level of success.

Adding new technology, new providers and new application creates an environment that is becoming exponentially complex. At the end, airlines being successful will definitively need to be able to make the right choices, reduce their risks and be carefully to limit their investment to solutions that will last. One of the factors that enables meeting these objectives and constrains is to share those risks and investments with industry partners. Another key element to choose adequate partners is the ubiquity of the solution they propose. As airlines fly everywhere, the solution chosen must be available globally. Solutions that remain only available in certain geographic location may certainly last in a specific market, but have not the potentials to become industry standards.

\section{Regulatory aspects}

A number of regulatory requirements and actors come into action when we talk about aircraft communications.

- Operating an access network generally imposes the use of radio licenses

- Dealing with ATS communications implies to interact with national ANSPs as customers or as providers/partners

- And many others

The ability to deal with such entities is a prerequisite to global service provisioning. Of course, but this is a special item, an aircraft embedded system needs to be certified at appropriate assurance level

\section{Vertical and Horizontal integration}

It is interesting to focus on the positioning of the success players in datalink history and see how things are evolving. Horizontal integration at access network and network level is compulsory to provide consistent services, and add value to the fact of integrating multiple dissimilar access networks (with their incumbent complexity due to the multiplicity of operators).

This is what happened in ACARS and ATN. Traditional DSPs started developing with vertically integrated solution (VHF - ACARS - some application services) to horizontal integration to make the services available worldwide (VHF operated by DSP and other access network services outsourced (Inmarsat). Traditional DSPs have positioned themselves more in the Cockpit communications domains than on cabin domain.

Another important factor here is the existence of historic operators/compulsory operators that are imposed by local regulations. The ability to deal with such entities is a prerequisite to global service provisioning. 
We could define vertical integration by the fact of acquiring the ability to control parts or all the actors/functions needed to provide an overall service, i.e. providing at the same time different levels of service (access, network, application, etc.), while horizontal integration could be defined as the fact of acquiring the ability to widen geographically or in terms of market target (e.g. cabin versus cockpit) a given service.

Figure 10 illustrates this concept. It is interesting to see that, depending on the market segment (ATS/AOC legacy,...), and the service level, the position of existing bridges (similar products that can satisfy upper services) can vary. For example, it is obvious to notice that ATS/AOC and EFB will likely call similar skills and services (IT integration, data production and publishing), while communication means between EFB and cabin could be shared (e.g. SBB,...).

ATS/AOC future ATS/AOC legacy EFB Cabin/Pax

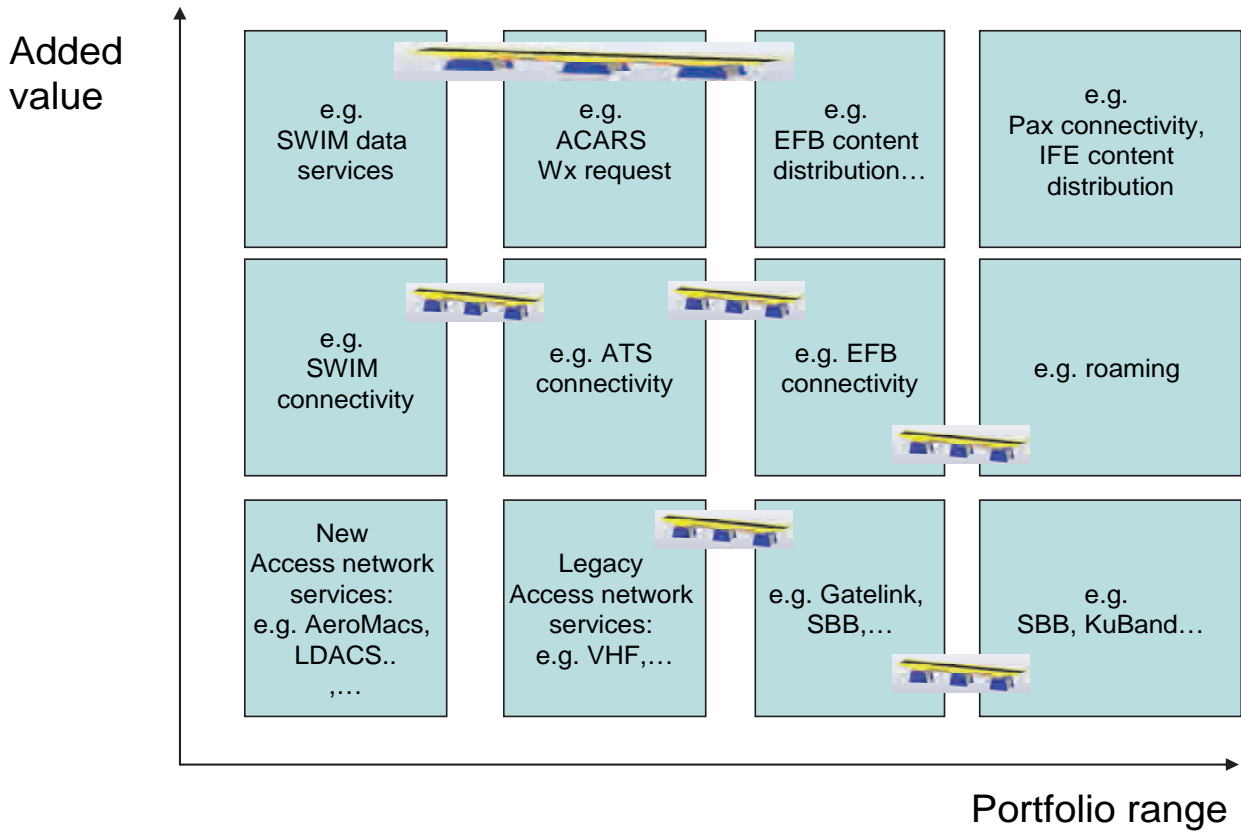

Fig. 10. Illustration of Vertical and horizontal integration. 


\section{Current and future NGA operator's views}

The recent survey with NGA operator mentioned earlier includes many indications that confirms and support many of the information given in this chapter. Here is a small overview of what some of the key players in airline operation are mutually saying about using new IT technologies in aircraft operation:

- Understanding the complexity and inter-relationship that will shape the future of aircraft operation result in a long learning curve.

- The requirement for cross organisational collaboration is viewed as an essential element for a successful program to implement new technologies for aircraft operation.

- Many delays are caused by the needs for common understanding and alignment of the multiple parties involved, including regulatory authority, airframers and standard bodies.

- Obsolescence of chosen technology in contrast to the life time of fleet-wide implementations is a major concern an often a road block to making technological choices.

- There is a tendency to make incremental steps forward as the solutions and industry vision evolves.

- The search for real business value leading to a successful business case is a difficult task in the current context.

\section{Conclusion}

Airlines expect to be able to meet their short and long term business objectives using the new IT technology available in the aircraft cockpit. No specific solution, IP broadband communication method or technology as yet rises to become the industry standard necessary to limit the risk associated with large deployment project. This is the case for both airlines and service providers. Legacy system and communication technology installed in today's aircraft will remain pertinent for the foreseen future and need to be integrated in the offered IT solutions. The complexity associated with the installation and operation of new IT systems is continuously rising. Absolute confidence in watertight security of the new systems and communication links must be achieved.

As the technologies used in aviation applications move from purpose-built to generic, the entry barriers for new entrants have been considerably lowered. Consequently the complexity and diversity of the solutions and required inter-relationship of the industry players is considerably augmented. Providers have to be carefully chosen by airlines based on their offer of valuable and compelling service that can assist them to make the most efficient use of their modern aircraft without compromising their operational flexibility or security. Solutions that can be built to take into consideration the various technology choices, requirements for global availability, the typical aircrafts projects life-time, integration with legacy systems and needs for common approaches will certainly have a better chance to be successful in the long term.

As we have seen above, from the customer's prospective, dealing with major partners providing horizontally integrated solutions, especially at access network level, will likely be the way to go, providing that the investment stays reasonable and offers an interesting return on investment scheme. Of course, horizontal integration should target the pertinent access network technologies (efficient, reasonable cost) that can be deployed on aircraft at 
reasonable cost and cycle. Each customer's case is specific, so it is of course too simplistic to summarize it this way, but this trend might well prove to be true.

It will be several years before new cockpit technology deliver on its expected benefits to reduce overall fleets operational cost and improve productivity, but we are clearly heading in that direction.

\section{Possible customers' perspective}

We do not take much risk if we say that customers seek

- Low cost

- $\quad$ SLA/SLO and adequate performances

- Globally available service - at least on strategic routes or locations

- If possible, end to end managed service

- And now, proper integration in their operations process (integration in their IT environment or hosted IT environment)

For some specific discriminating services towards their competition, some airlines may be willing to invest to offer unique services to attract new passengers, for example in the domain of aircraft passenger services.

We could conclude from this chapter that, in order to make future connectivity services a success, the airlines will seek for service providers

- Horizontally integrated

- Multiple radio access networks and ground networks

- Vertically integrated

- Application services

- Up to the IT infrastructures

And of course there is a STRONG

- Need for competition

- Standardization

- Multiple players

This is a general conclusion, and, as said before, airlines needs need to be studied on a case by case basis, but we tried here to give general trends that will hopefully help the reader have a wider view of the situation.

\section{Appendix - case study: AeroMACS}

This section aims at introducing the various possible actors in AeroMACS connectivity service and identifies their possible contractual relationship.

The following applications have been identified as target by RTCA SC223 and/or Eurocae WG82:

- $\quad$ Fixed users (RTCA only)

- $\quad$ Airport LAN extensions

○ Unique equipment (terminals, cameras,...)

- Or Multiple equipment behind Mobile System (MS)

- $\quad$ ANSP managed equipment

○ Integration of RNAV systems, radar... into ANSP network

- Mobile users 
- $\quad$ Airport trucks (catering, maintenance, fuel....)

- Luggage terminals,....

- Single users (= user terminal) belonging to different networks or LANs (!) (airport, $\mathrm{MRO}$, catering, fuel......)

- $\quad$ Support for VoIP (RTCA only)

- Aircraft

- $\quad$ ATC, AOC,direct operational impact / safety impact

- AAC applications no direct operational impact

- End to end (to airline and ANSPs) communication but also potentially local communications (cache servers)

- Need to segregate on aircraft at minimum between various users domain (avionics (ATC, AOC) - IT domain (AOC, AAC) - Pax domain)

\subsection{Actors and possible business / contractual relationship}

WiMax forum (WMF) Network architecture group has identified the following typical business relationship for WiMax as shown in Figure 11.

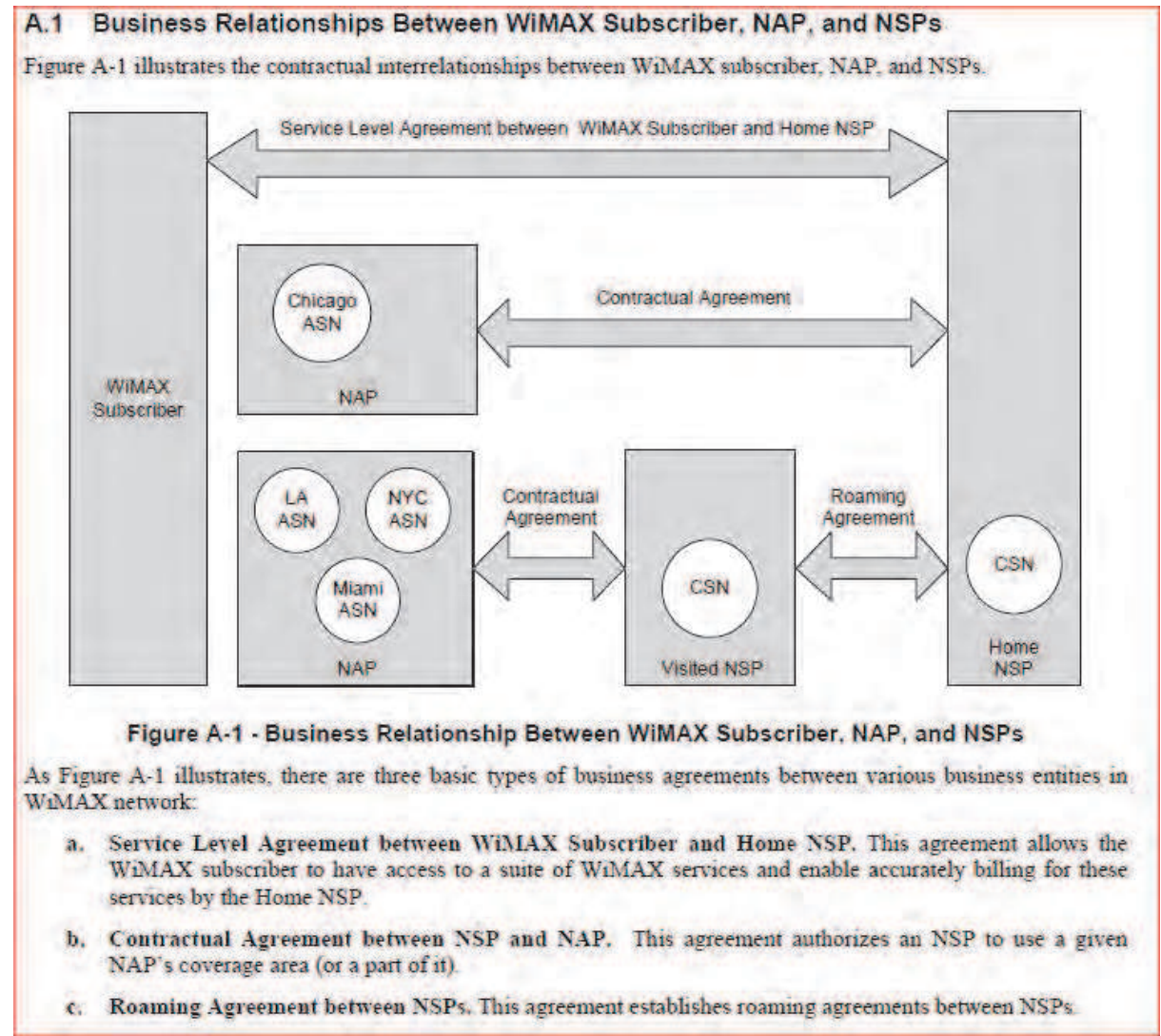

Fig. 11. WiMax forum identified actors and relationship. 
In the aeronautical environment, the following actors have been identified:

- NAP

- $\quad$ Specialized Airport operator

- Traditional DSP (ARINC, SITA, AVICOM,....)

- ANSP

- $\quad$ V-NSP

- $\quad$ Traditional DSP (ARINC, SITA, AVICOM,....)

- $\quad$ ANSP

- Local operator

- $\quad$ Specialized Airport operator

- H-NSP

- $\quad$ Traditional DSP (ARINC, SITA, AVICOM,....)

- Others

In Figure 12, the reference contractual/business relation ship between various actors in the aeronautical environment could be as illustrated.

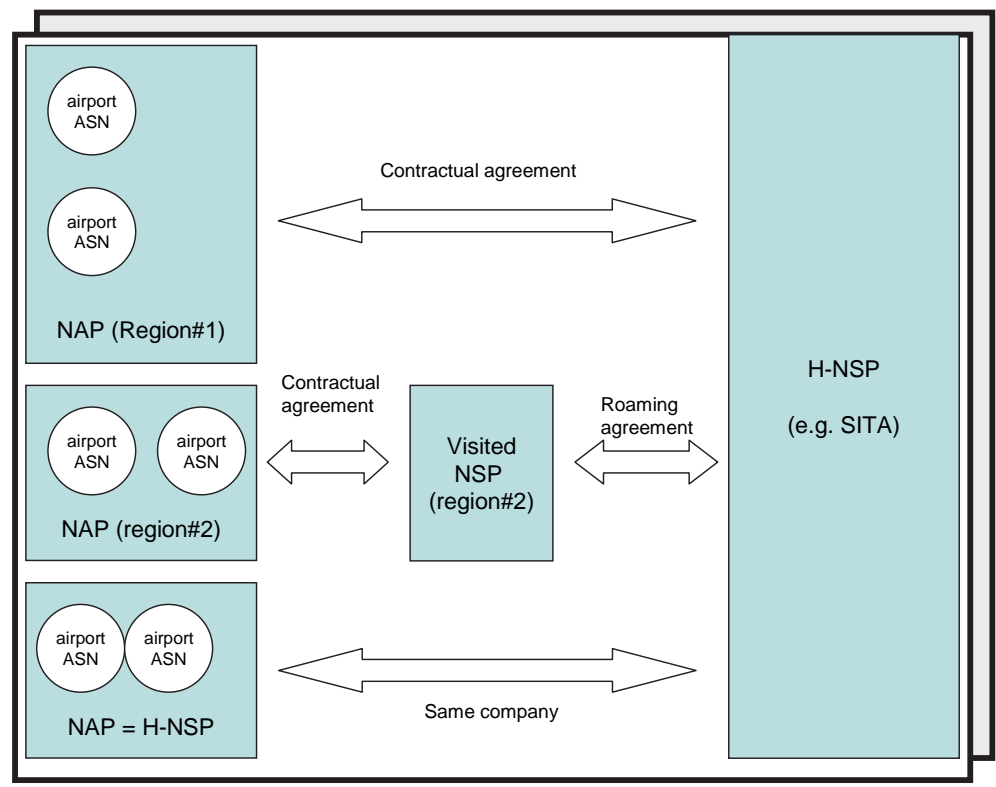

\section{Service}

Level

Agreement

Between wimax

operator and

Home NSP

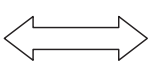

Airline

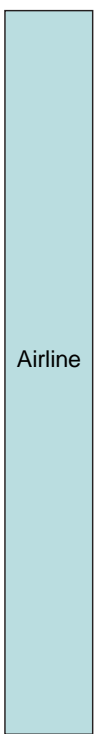

Fig. 12. Actors and possible relationship for Aeromacs. 


\subsection{Network deployment use cases}

The following deployment use cases have been identified by WMF (reference).:

- $\quad$ A.3.1 NAP Sharing by Multiple NSPs

- A.3.2 Single NSP Providing Access Through Multiple NAPs

- $\quad$ A.3.3 Greenfield WiMAX NAP+NSP

- $\quad$ A.3.4 Greenfield WiMAX NAP+NSP with NAP Sharing

- $\quad$ A.3.5 Greenfield WiMAX NAP+NSP Providing Roaming

- $\quad$ A.3.6 Visited NSP Providing WiMAX Services

- $\quad$ A.3.7 Home NSP Providing WiMAX Services

The deployment use cases identified above are instantiated for aeronautical environment as follows - please note that multiple use cases will be supported at the same time. Especially, an aircraft will need to be able to interact with various use cases, depending of its location at time $\mathrm{T}$.

Open points to be discussed:

- Multiple NAPs will be available in a given airport and one used at a given time by an aircraft

- An aircraft may contract several H-NSPs and selection will be done in real time.

- Need for NAPs to advertise supported NSPs (H-NSP??) and the aircraft will then select the preferred H-NSP

\subsection{RF deployment use cases}

Several radio / NAP deployments are possible:

1. Single NAP - single radio channel

- Use service flows / QoS to distinguish between application types (aircraft)

- $\quad$ All NSPs advertise on this channel

2. Single NAP - Multiple radio channels

- 1 channel for aircraft communications

- All NSPs advertise on this channel

- 1 or several channel for fixed and mobile users

3. Single NAP - Multiple radio channels

- 1 channel for safety communications (ATC/AOC) for aircraft and mobiles

- 1 channel for non safety (AAC), including mobiles

- 1 channel for fixed users (RTCA use case)

- Implies 2 radios on aircraft. This solution is probably not adequate due to aircraft systems complexity

4. Multiple NAPs - Multiple radio channels (NAP+NSP solution)

- 1 several channels for fixed and mobile users

Various constraints need to be taken into account to determine the appropriate solution(s):

- Channels allocation scheme selected by various states/countries

- Limit configuration changes on aircraft side

- Segregation between non safety/dispatch impacting traffic and non safety / non dispatch impacting traffic

- An aircraft will need to interoperate with any AeroMACS infrastructure, while ground mobiles and fixed users may be tailored to specific ground infrastructures

- $\quad$ Fixed infrastructures support safety traffic may need to be severely segregated from mobile infrastructures 

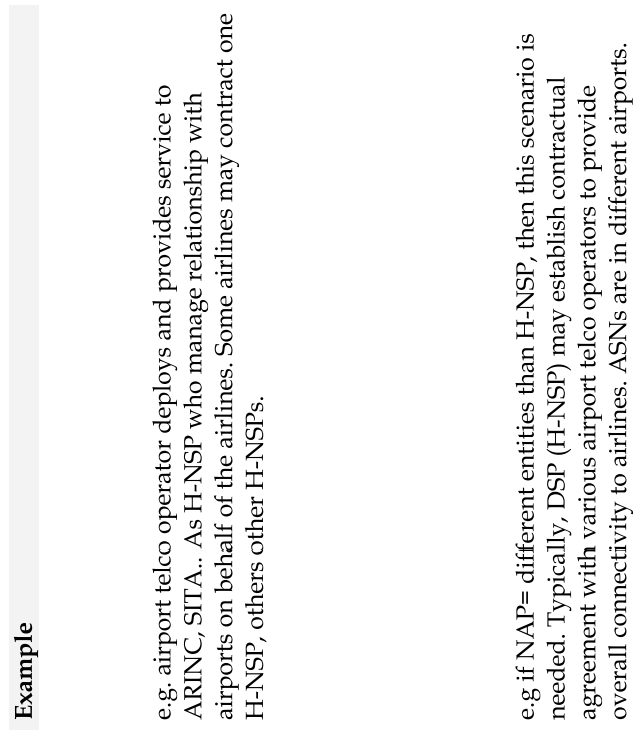

후욤

㐘

ปี

岕

홍

흥 흥

웜

ส

जै

垔

党䎡

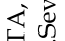

氙客

की

出 寻

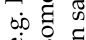
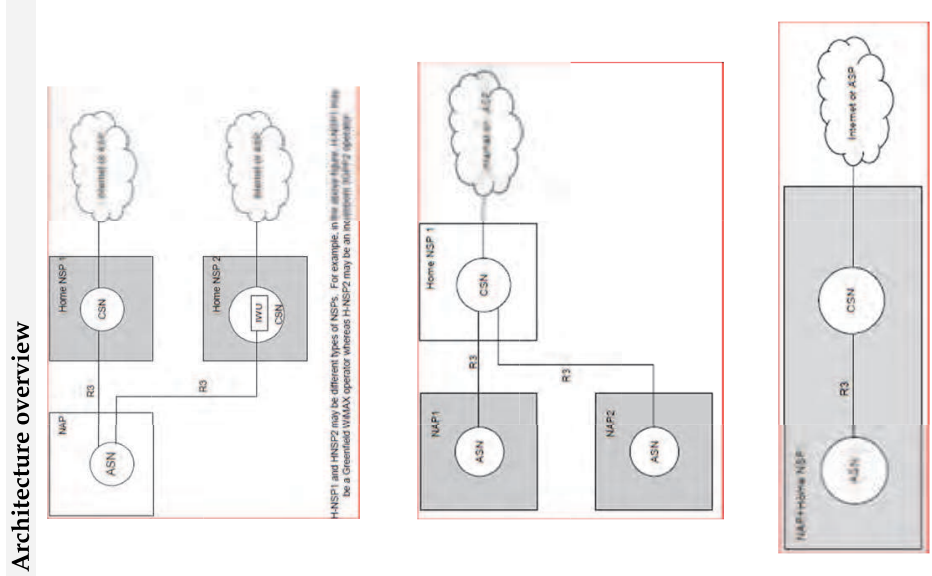

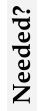

$\stackrel{\infty}{2}$

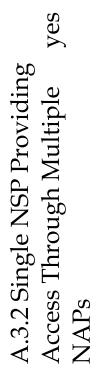

$\stackrel{\infty}{x}$
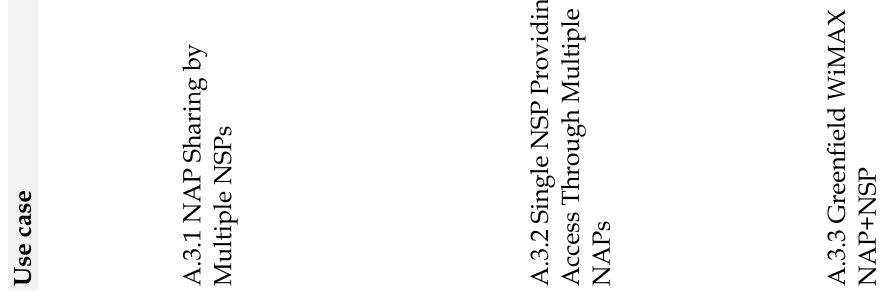

Table 2. Network deployment use cases (part I). 

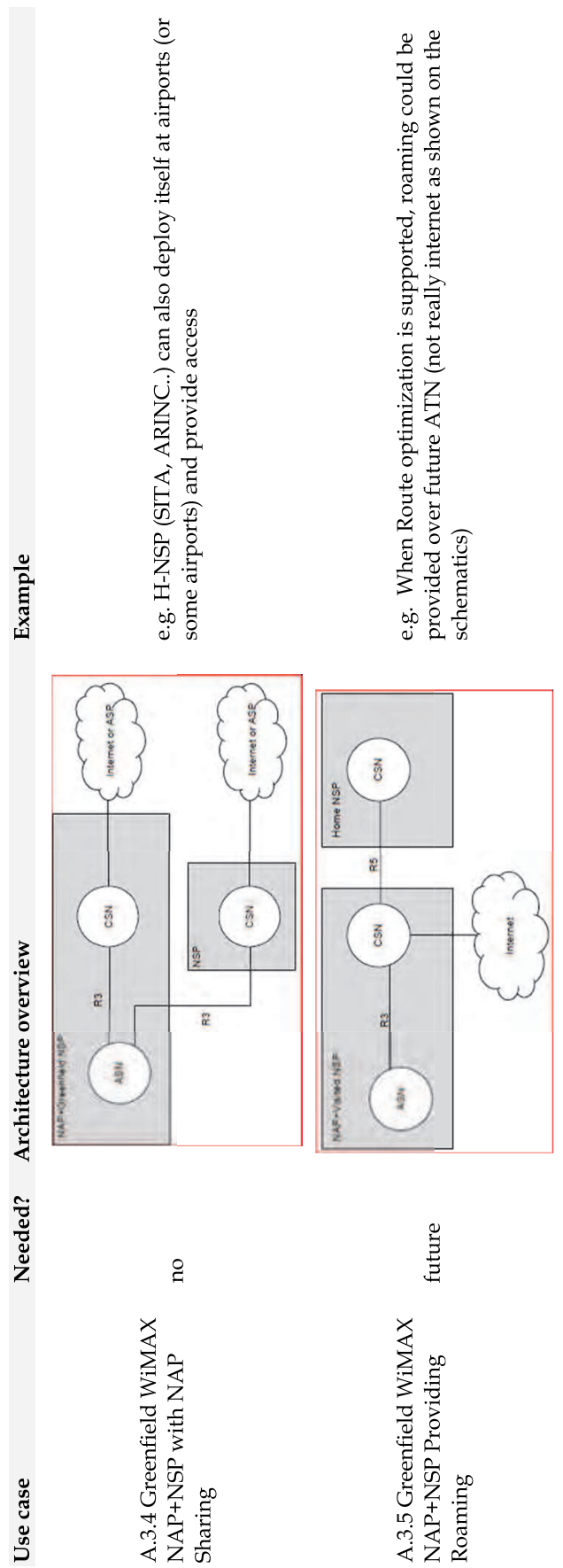

Table 2. Network deployment use cases (part II). 

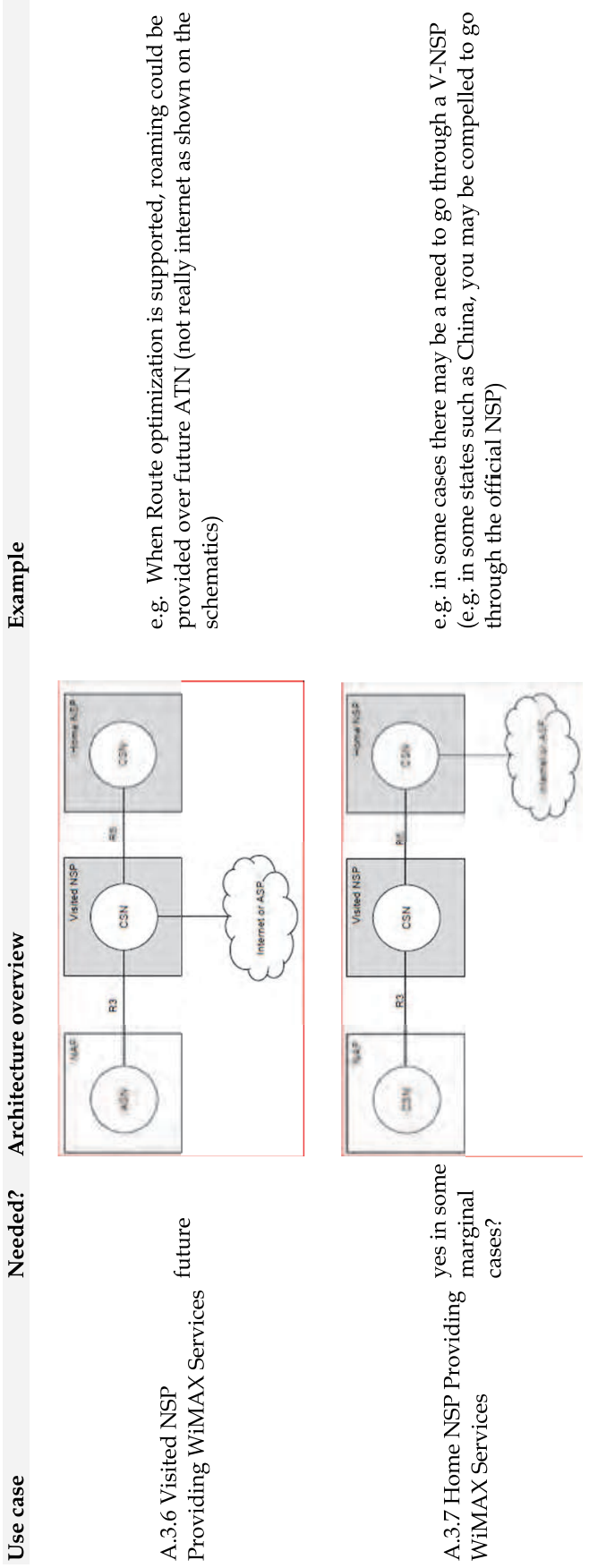

Table 2. Network deployment use cases (part III). 


\subsection{Conclusion on the AeroMACS case study}

We have seen that many deployment configurations were possible for AeroMACS connectivity service. The deployment / contractual relationships and associated actors are still under discussion in various standardization bodies. However, we can see that the number of actors and the variety of situations will likely drive the need, as seen in legacy/emerging connectivity services, for overall mobility service providers, aka Global DSPs, to hide the complexity of the connectivity service schemes to be establish to provide a global, seamless, simple and efficient service to the various service customers.

\section{Acknowledgement}

The research leading to these results has been partially funded by the European Community's Seventh Framework Programme (FP7/2007-2013) under Grant Agreement $\mathrm{n}^{\circ}$ 233679. The SANDRA project is a Large Scale Integrating Project for the FP7 Topic AAT.2008.4.4.2 (Integrated approach to network centric aircraft communications for global aircraft operations). The project has 31 partners and started on 1st October 2009.

\section{References}

WiMax Forum Network Architecture (Stage 2: Architecture Tenets, Reference Model and Reference Points) [Part 3 - Informative Annex] Release 1.0 Version 4

ICAO ANNEX 10 to the Convention on International Civil Aviation Aeronautical Telecommunications (Volumes I, II, III, IV and V)

SITA AIRCOM new generation services Positioning Paper - White Paper - 2010

SITA - The "Digital aircraft" -heralding a new generation of aircraft operations - New Frontiers paper - 2010

WiMax Forum Network Architecture (Stage 2: Architecture Tenets, Reference Model and Reference Points) [Part 0] Release 10 Version 4

(WMF-T32-001-R010v04_Network-Stage2-Part0_0)

WiMax Forum Network Architecture (Stage 2: Architecture Tenets, Reference Model and Reference Points) [Part 1] Release 10 Version 4

WMF-T32-002-R010v04_Network-Stage2-Part1_2_0

WiMax Forum Network Architecture (Stage 2: Architecture Tenets, Reference Model and Reference Points) [Part 2] Release 10 Version 4

WMF-T32-003-R010v04_Network-Stage2-Part2 


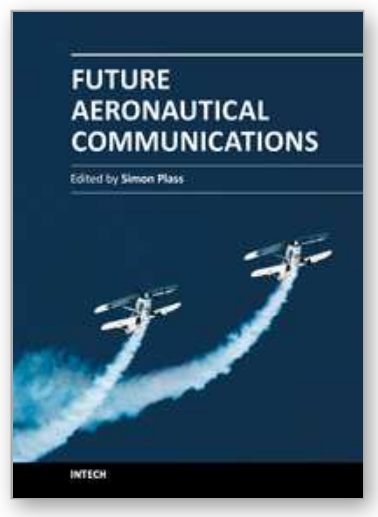

\author{
Future Aeronautical Communications \\ Edited by Dr. Simon Plass
}

ISBN 978-953-307-625-6

Hard cover, 378 pages

Publisher InTech

Published online 26, September, 2011

Published in print edition September, 2011

There are well-founded concerns that current air transportation systems will not be able to cope with their expected growth. Current processes, procedures and technologies in aeronautical communications do not provide the flexibility needed to meet the growing demands. Aeronautical communications is seen as a major bottleneck stressing capacity limits in air transportation. Ongoing research projects are developing the fundamental methods, concepts and technologies for future aeronautical communications that are required to enable higher capacities in air transportation. The aim of this book is to edit the ensemble of newest contributions and research results in the field of future aeronautical communications. The book gives the readers the opportunity to deepen and broaden their knowledge of this field. Todayâ $€^{\mathrm{TM}} \mathrm{S}$ and tomorrowâ€ $€^{\mathrm{TM}} \mathbf{S}$ problems / methods in the field of aeronautical communications are treated: current trends are identified; IPv6 aeronautical network aspect are covered; challenges for the satellite component are illustrated; AeroMACS and LDACS as future data links are investigated and visions for aeronautical communications are formulated.

\title{
How to reference
}

In order to correctly reference this scholarly work, feel free to copy and paste the following:

Frederic Durand and Luc Longpre (2011). Handling Transition from Legacy Aircraft Communication Services to New Ones - A Communication Service Provider's View, Future Aeronautical Communications, Dr. Simon Plass (Ed.), ISBN: 978-953-307-625-6, InTech, Available from: http://www.intechopen.com/books/futureaeronautical-communications/handling-transition-from-legacy-aircraft-communication-services-to-new-ones-acommunication-service-

\section{INTECH}

open science | open minds

\section{InTech Europe}

University Campus STeP Ri

Slavka Krautzeka 83/A

51000 Rijeka, Croatia

Phone: +385 (51) 770447

Fax: +385 (51) 686166

www.intechopen.com

\section{InTech China}

Unit 405, Office Block, Hotel Equatorial Shanghai

No.65, Yan An Road (West), Shanghai, 200040, China 中国上海市延安西路65号上海国际贵都大饭店办公楼 405 单元

Phone: +86-21-62489820

Fax: +86-21-62489821 
(C) 2011 The Author(s). Licensee IntechOpen. This chapter is distributed under the terms of the Creative Commons Attribution-NonCommercialShareAlike-3.0 License, which permits use, distribution and reproduction for non-commercial purposes, provided the original is properly cited and derivative works building on this content are distributed under the same license. 